\title{
NULL QUADRATURE DOMAINS AND A FREE BOUNDARY PROBLEM FOR THE LAPLACIAN
}

\author{
LAVI KARP* AND AVMIR S. MARGULIS \\ Dedicated to Harold S. Shapiro
}

\begin{abstract}
Null quadrature domains are unbounded domains in $\mathbb{R}^{n}(n \geq 2)$ with external gravitational force zero in some generalized sense. In this paper we prove that the complement of null quadrature domain is a convex set with real analytic boundary. We establish the quadratic growth estimate for the Schwarz potential of a null quadrature domain which reduces our main result to Theorem II of [8] on the regularity of solution to the classical global free boundary problem for Laplacian. We also show that any null quadrature domain with non-zero upper Lebesgue density at infinity is half-space.
\end{abstract}

\section{INTRODUCTION}

An open set $\Omega$ in $\mathbb{R}^{n}$ is called a null quadrature domain if

$$
\int_{\Omega} h d x=0
$$

for all harmonic and integrable functions $h$ in $\Omega$. This class of domains includes half-spaces, exterior of ellipsoids, exterior of strips, exterior of elliptic paraboloids and cylinders over domains of these types. In 1980 Sakai showed that in $\mathbb{R}^{2}$ any null quadrature domain belongs to one of the categories above [35]. A complete description of all null quadrature domains in higher dimensions has remained an open problem; the best result was obtained in [25], where the case of a complement contained in a cylinder of co-dimension two was settled.

An equivalent definition of null quadrature domain $\Omega$ is by means of a free boundary problem and potentials. If there exists a solution $u$ to

2010 Mathematics Subject Classification. Primary 35J25, 31B20; Secondary $35 \mathrm{~B} 65,31 \mathrm{C} 15$.

Key words and phrases. A free boundary problem, null quadrature domains, Schwarz potential, quadratic growth.

*Supported by ORT Braude College's Research Authority. 
the system

$$
\Delta u=-\chi_{\Omega}, \quad u=|\nabla u|=0 \text { on } \mathbb{R}^{n} \backslash \Omega,
$$

then $u$ is called the Schwarz potential of $\Omega$. The Schwarz potentials of null quadrature domains are a subclass of all solutions to (1.1) (see Section 3). This subclass is characterized by an additional condition, namely, the Schwarz potential $u$ is a (Newtonian) potential of $\Omega$, and it can be expressed in term of a growth property at infinity. Thus a domain $\Omega$ is a null quadrature domain if and only if there exists a solution $u$ to the free boundary problem (1.1) satisfying

$$
u(x)=o\left(|x|^{3}\right) \quad \text { as }|x| \rightarrow \infty,
$$

see Corollary 3.2 below.

Shapiro conjectured that the complement of a null quadrature domain is a convex set [39], and he showed it under some additional restrictions both on the domain $\Omega$ and its Schwarz potential $u$.

Under the assumption that the Schwarz potential $u$ of $\Omega$ has a quadratic growth, Caffarelli, Karp and Shahgholian proved that the complement, $\Omega^{c}$, is convex and the boundary, $\partial \Omega$, is a real analytic surface, [8, Theorem II].

Our principal result confirms Shapiro's conjecture and shows that any null quadrature domain $\Omega$ has a convex complement and real analytic boundary. The main technical tool is the establishment of the quadratic growth of solutions to the free boundary problem (1.1) when the a priori growth condition (1.2) is imposed.

The system (1.1) arises also in the investigation of the regularity of free boundaries in the obstacle problem as a "blow up limits' (see e.g. $[6,14])$. However, in that case there is an additional condition, namely $u \leq 0$ in $\mathbb{R}^{n}$. Under this condition, Caffarelli proved that the complement of $\Omega$ is a convex set [6]. The essence of Theorem II in [8] is showing that any Schwarz potential with a quadratic growth is nonpositive, hence the unsigned free boundary problem inherits the same regularity properties as the obstacle problem in that case. However, when the growth condition (1.2) is removed, then there are solutions to (1.2) with a non-convex complement, see [39] and Section 3 for further examples.

The quadratic growth problem occurs also in the local version of (1.1), that is, if $x_{0}$ is a point on the free boundary, then a priori $|u(x)| \leq$ $C\left|x-x_{0}\right|^{2} \log \left|x-x_{0}\right|$. In that case the quadratic growth was resolved in [8, Theorem I]. In order to obtain it in the global case, we combine the techniques of [8] with Shapiro's quasi-balayage [42], and some estimates of the generalized Newtonian potential [25]. 
The generalized Newtonian potential is a multi-valued right inverse to the Laplacian and unique up to a harmonic polynomial of degree not exceeding two. It enables the computation of a potential of arbitrary domains in $\mathbb{R}^{n}$ in a similar manner to the ordinary Newtonian potential (see $[22,25,28]$ ). For example, the distribution $u$ which satisfies (1.1) and (1.2) is a generalized Newtonian potential of the null quadrature domain $\Omega$. Since $u$ is zero on $\Omega^{c}$, we see that null quadrature domains consist of open sets in $\mathbb{R}^{n}$ having zero gravitational force in their complement.

Another characterization of null quadrature domains is through the potential of their complement. Namely, $\Omega$ is a null quadrature domain if and only if the generalized Newton potential of $D:=\mathbb{R}^{n} \backslash \Omega$ coincides with a quadratic polynomial in $D$ (see Theorem 2.6 below). In case $D$ is bounded, this means that in a suitable coordinates system the shell $\lambda D \backslash D$ produces no gravitational force in the cavity $D$. The classification of such domain was settled by Dive [13], and Nikliborc [31]. We refer to [30] for further discussion on that property (see also $[15,24,25])$.

Thus the main difficulty of the classification of null quadrature domains occurs when the complement $D:=\mathbb{R}^{n} \backslash \Omega$ is unbounded. In this paper we show that if $D$ has a positive upper Lebesgue density of at infinity, then it must be a half-space. The classification when the Lebesgue density of $D$ is zero will be discussed in [30].

The growth condition (1.2) is indispensable for $\Omega$ being a null quadrature domain. Indeed, Gustafsson and Shapiro [39], and independently Richardson [34], used conformal mappings in order to construct a two dimensional domain $\Omega$ and its the Schwarz potential $u$, such that $\mathbb{R}^{2} \backslash \Omega$ is bounded and $u$ has polynomial growth, or even an essential singularity, at infinity. Hence the Scwharz potential $u$ is not a (Newtonian) potential and consequently the complement $\mathbb{R}^{2} \backslash \Omega$ is not necessarily convex. Shapiro raised the question whether an analogous example exists in higher dimensions [39]. We present an $n$-dimensional version of the above example, our construction is based upon a theorem on the solvability "in the small" of the inverse problem of potential theory (see [20] for details).

The plan of the paper is as follows. In the next Section we provide some background on generalized Newtonian potential, quadrature domains and the Schwarz potentials. Section 3 deals with harmonic continuation of potentials, its relations to null quadrature domain and the construction of a solution to (1.1) with arbitrarily growth at infinity. In the last section we prove the quadratic growth of the Schwarz potential and discuss geometric properties of null quadrature domains. 


\section{BACKGROUND AND NOTIONS}

In this section we present a brief account of the generalized Newtonian potential, quadrature domains and Schwarz potentials. Throughout of this paper points in $\mathbb{R}^{n}$ are denoted by $x$ or $y,(x \cdot y)$ is the scalar product and $|x|=\sqrt{(x \cdot x)}$. For a set $A \subset \mathbb{R}^{n}$, we denote by $A^{c}$ the complement of $A, \bar{A}$ the closure of $A, \operatorname{int}(A)$ its interior, $\partial A$ the boundary of $A, \chi_{A}$ the characteristic function of $A$ and by $|A|$ is the Lebesgue measure of a measurable set $A$. An open ball with radius $r$ and center $x_{0}$ is denoted by $B_{r}\left(x_{0}\right)$.

2.1. Generalized Newtonian Potential. The definition and establishment of basic properties of this potential were carried out in [25] (see also $[22,28])$. Here we recall the definition and present a few applications. A different extension of the Newtonian potential for measures with non-compact support is given in [11, Ch.II, §3]. But their extension does not include the class of densities in $L^{\infty}\left(\mathbb{R}^{n}\right)$.

The Newtonian potential of a measure $\mu$ with compact support is defined by means of the convolution

$$
V(\mu)(x)=(J * \mu)(x)=\int J(x-y) d \mu(y),
$$

where

$$
J(x)= \begin{cases}-\frac{1}{2 \pi} \log |x|, & n=2 \\ \frac{1}{(n-2) \omega_{n}|x|^{n-2}}, & n \geq 3\end{cases}
$$

and $\omega_{n}$ is the area of the unit sphere in $\mathbb{R}^{n}$. The potential $V(\mu)$ satisfies the Poisson equation $\Delta V(\mu)=-\mu$ in the distributional sense. The generalized Newtonian potential is a multi-valued right inverse of the Laplacian on the space $\mathcal{L}$, the space of all Radon measures $\mu$ in $\mathbb{R}^{n}$ satisfying condition

$$
\|\mu\|_{\mathcal{L}}:=\int \frac{d|\mu|(x)}{1+|x|^{n+1}}<\infty .
$$

The linear space $\mathcal{L}$ is the Banach space with the norm defined by (2.2). For $\mu \in \mathcal{L}$ we define the third order generalized derivatives of the potential as tempered distributions by setting

$$
\left\langle V^{\alpha}(\mu), \varphi\right\rangle:=-\int \partial^{\alpha} V(\varphi)(x) d \mu(x), \quad \varphi \in \mathcal{S},|\alpha|=3,
$$

where $\mathcal{S}$ is the Schwartz class of rapidly decreasing functions. 
Definition 2.1. The generalized Newtonian potential $V[\mu]$ of a measure $\mu \in \mathcal{L}$ is the set of all solutions to the system

$$
\left\{\begin{array}{l}
\Delta u=-\mu \\
\partial^{\alpha} u=V^{\alpha}(\mu), \quad|\alpha|=3 .
\end{array}\right.
$$

The existence of solutions to system (2.3) was proved in [25]. The solution of system (2.3) is unique modulo $\mathcal{H}_{2}$, the space of all harmonic polynomials of degree at most two. The operator $V: \mathcal{L} \rightarrow \mathcal{S}^{\prime} / \mathcal{H}_{2}$ is continuous [25].

Here and in the sequel we denote by $V[\sigma]$ the generalized potential of a measure $\mu=\sigma \lambda$, where $\lambda$ is the Lebesgue measure and $\sigma \in L^{\infty}\left(\mathbb{R}^{n}\right)$. We use the notation $V[A]=V\left[\chi_{A}\right]$ when $\sigma=\chi_{A}$, the characteristic function of a measurable set $A$. For $v \in V[\mu]$, we may use the terminology of a generalized Newtonian potential of $\mu$, or simply a potential of $\mu$.

The generalized Newtonian potential is an adequate notion for explicit calculations of potentials of unbounded domains and of measures with a non-compact support. The following two examples demonstrate it. By Definition 2.1,

$$
V\left[\mathbb{R}^{n}\right]=-\frac{|x|^{2}}{2 n}+\mathcal{H}_{2},
$$

and hence for any measurable set $A$ we have the complementary formula

$$
V[A]+V\left[A^{c}\right]=-\frac{|x|^{2}}{2 n}+\mathcal{H}_{2} .
$$

In [25] we derived an explicit formula for the potential of an arbitrary cone. Let $K$ be a cone with the vertex at the origin. Then $u$ is a generalized Newtonian potential of the cone $K$ if and only if

$$
u(x)=P_{2}(x) \log |x|+\Phi(x)+q(x),
$$

where $P_{2}$ is a homogeneous harmonic polynomial of degree two, $\Phi$ is a homogeneous function of order two and $q$ is a linear polynomial. If $P_{2} \not \equiv 0$, then the logarithmic term is the dominant term both at the origin and infinity. If $K$ is convex and different from a half-space, or if $\bar{K}$ is contained in a "critical cone"

$$
K_{c r}:=\left\{x:|x|^{2}<n(x \cdot a)^{2}\right\},
$$

where $a$ is a unit vector, then $P_{2} \not \equiv 0$, i.e. the logarithmic term is not zero. On the other hand, for any $\varepsilon>0$ there are cones $K \subset\left\{x:|x|^{2}<\right.$ $\left.(n+\varepsilon)(x \cdot a)^{2}\right\}$ with $P_{2} \equiv 0$. 
Another useful feature is an integral representation of the generalized potential [22, 25]. Let

$$
J_{2}(x, y):=J(x-y)-\chi_{\{y:|y|>1\}} \sum_{|\alpha| \leq 2} \frac{(-x)^{\alpha}}{\alpha !} \partial^{\alpha} J(y)
$$

and set

$$
V_{2}(\mu)(x):=\int_{\mathbb{R}^{n}} J_{2}(x, y) d \mu(y) .
$$

Then the potential $V_{2}(\mu)$ belongs to $V[\mu]$ (cf. [25]), and for any $\sigma \in$ $L^{\infty}\left(\mathbb{R}^{n}\right)$ the following estimate holds (cf. [22, 41]):

$$
\left|V_{2}(\sigma)(x)\right| \leq C\|\sigma\|_{\infty}(1+|x|)^{2} \log (2+|x|) .
$$

Note that any $v \in V[\sigma]$ must satisfy (2.8), since $V[\sigma]=V_{2}(\sigma)+\mathcal{H}_{2}$. Therefore, by Liouville's theorem we get an equivalent definition of the generalized Newtonian potential for $\sigma \in L^{\infty}\left(\mathbb{R}^{n}\right)$ :

Proposition 2.2. A solution $u$ to the Poisson equation $\Delta u=-\sigma$ with $\sigma \in L^{\infty}\left(\mathbb{R}^{n}\right)$ is a generalized potential of $\sigma$ if and only if it satisfies the estimate (2.8).

\subsection{Quadrature Domains and Schwarz potentials.}

The mean value theorem for harmonic functions can be written as a quadrature identity

$$
\int_{\Omega} h d x=|\Omega| h\left(x_{0}\right)=\int h d \mu,
$$

where $\Omega=B_{r}\left(x_{0}\right)$ is a ball centered at $x_{0}$ and $\mu$ is the Dirac measure times the volume of the ball. This property is equivalent to Newton's theorem asserting that the ball gravitationally attracts points outside it as if all its mass were concentrated at the center of the ball.

A natural generalization of the above property of balls is the notion of quadrature domain (see [17, 36, 38]):

Definition 2.3. Let $\mu$ be a Radon measure in $\mathbb{R}^{n}$. An open set $\Omega$ is called a quadrature domain of $\mu$ and with respect to harmonic functions if $\left.\mu\right|_{\mathbb{R}^{n} \backslash \Omega}=0$, and

$$
\int_{\Omega} h d x=\int h d \mu \quad \text { for all } h \in H L^{1}(\Omega)
$$

where $H L^{1}(\Omega)$ is the class of all functions harmonic in $\Omega$ and integrable over $\Omega$. 
The identity of the external potential of $\Omega$ and $\mu$ out of $\Omega$ is probably one of the most important property of a quadrature domains.

Observe, if $\Omega$ is a quadrature domain of $\mu$ and $\bar{\Omega} \neq \mathbb{R}^{n}$, then we may assume that $B_{1}(0) \subset \Omega^{c}$. Hence for any $y \in \Omega$, the kernel $J_{2}$ defined by (2.6) becomes

$$
J_{2}(x, y)=J(x-y)-\sum_{|\alpha| \leq 2} \frac{(-x)^{\alpha}}{\alpha !} \partial^{\alpha} J(y) .
$$

Therefore for any fixed $x \in \Omega^{c}$ the function $J_{2}(x, \cdot)$ and its first order derivatives belong to $H L^{1}(\Omega)$. Applying the identity (2.9) to these functions, we conclude that

$$
\left.V_{2}(\Omega)\right|_{\Omega^{c}}=\left.V_{2}(\mu)\right|_{\Omega^{c}} \text { and }\left.\nabla V_{2}(\Omega)\right|_{\Omega^{c}}=\left.\nabla V_{2}(\mu)\right|_{\Omega^{c}}
$$

Hence, $u:=V_{2}(\Omega-\mu)$ is the solution of the following free boundary problem:

$$
\left\{\begin{array}{lll}
\Delta u=-\left(\chi_{\Omega}-\mu\right) & \text { in } & \mathbb{R}^{n} \\
u=|\nabla u|=0 & \text { in } & \mathbb{R}^{n} \backslash \Omega
\end{array} .\right.
$$

Obviously, if $\bar{\Omega} \neq \mathbb{R}^{n}$, then the solution of (2.10) is unique. This follows from the fact that the difference of any two solutions is a harmonic function in $\mathbb{R}^{n}$ which vanishes on $\Omega^{c}$.

Definition 2.4. A distribution u satisfying (2.10) is called the Schwarz potential of the pair $(\Omega, \mu)$ (in [41] it is called "the modified Schwarz potential").

Thus whenever $\Omega$ is a quadrature domain of a measure $\mu$, then the Schwarz potential of the pair $(\Omega, \mu)$ exists, moreover, in that case the Schwarz potential $u$ is a potential, that is, $u \in V[\Omega-\mu]$.

Therefore, in order that the existence of the Schwarz potential would imply that $\Omega$ is a quadrature domain, it is necessary that the Schwarz potential is a potential. But this is not sufficient. Indeed, in [25] we presented an example of a pair $(\Omega, \mu)$ with a bounded domain $\Omega$ and a finite measure $\mu$ such that $\left.\mu\right|_{\mathbb{R}^{n} \backslash \Omega}=0$ for which the Schwarz potential of $(\Omega, \mu)$ is a potential. But the space $H L^{1}(\Omega)$ is not contained in $L^{1}(|\mu|)$, so identity (2.9) cannot hold. Therefore there is a subtle difference between the property of an open set $\Omega$ being a quadrature domain of $\mu$, and the existence of the Schwarz potential of $(\Omega, \mu)$ which is a potential. Under certain restrictions, the equivalence does hold (see e.g. $[30, \S 2],[17,35])$.

Proposition 2.5. Let $\Omega$ be an open set and assume the measure $\mu$ has a compact support in $\Omega$ or $\mu=\sigma \lambda$, where $\sigma \in L^{\infty}\left(\mathbb{R}^{n}\right)$ and $\lambda$ is the 
Lebesgue measure. Then $\Omega$ is a quadrature domain of $\mu$ if and only if there exists $u \in V[\Omega-\mu]$ which is the Schwarz potential of $(\Omega, \mu)$.

The main theme of this paper is null quadrature domains, that is, identity (2.9) holds with $\mu \equiv 0$. By Proposition 2.5 this is equivalent to the existence of $u \in V[\Omega]$ satisfying (2.10). It means that the domain $\Omega$ produces zero gravitational force in the complement $\Omega^{c}$. Applying the complementary formula (2.4) to the generalized potential of a null quadrature domain $\Omega$, we obtain:

Theorem 2.6 ([22, 25]). An open set $\Omega$ is a null quadrature domain if and only if $V\left[\mathbb{R}^{n} \backslash \Omega\right]$ coincides with a quadratic polynomial in $\mathbb{R}^{n} \backslash \Omega$.

Remark 2.7. Newton discovered that the gravitational attraction at any internal point of elliptical shell is zero (see e.g. [9]). This phenomenon is equivalent to the property that the internal Newtonian potential of ellipsoids is a quadratic polynomial. Conversely, if $D$ is a bounded domain and its Newtonian potential coincides with a quadratic polynomial in $D$, then $D$ is an ellipsoid $[4,13,31]$. The characterization of domains $D$ for which their internal generalized Newtonian potential is a quadratic polynomial is an open problem.

We discuss now local properties of the Schwarz potential. Suppose $u$ is a Schwarz potential of $(\Omega, \mu)$ and $x_{0} \in \partial \Omega \backslash \operatorname{supp}(\mu)$. Then $u$ satisfies the free boundary problem

$$
\left\{\begin{array}{ll}
\Delta u=-1 & \text { in } \Omega \cap B_{r}\left(x_{0}\right) \\
u(x)=|\nabla u(x)|=0 & \text { on } B_{r}\left(x_{0}\right) \backslash \Omega
\end{array} .\right.
$$

Lemma 2.11 in [25] claims that under these conditions the free boundary $\partial \Omega \cap B_{r}\left(x_{0}\right)$ has zero Lebesgue measure (Actually this Lemma is valid in a much wider situations and it can be applied whenever the density of $\Omega$ is a positive $L^{\infty}$-function). Therefore, if $\Omega$ is a quadrature domain of a measure $\mu$ such that $\operatorname{supp}(\mu) \cap \partial \Omega=\emptyset$, then $\partial \Omega$ has zero Lebesgue measure and consequently $\Omega_{0}=\operatorname{int}(\bar{\Omega})$ satisfies the same quadrature identity (2.9). Therefore in the sequel we will assume that any quadrature domain $\Omega$ is the interior of its closure, and we may drop the requirement that the gradient of the Schwarz potential in (2.10), vanishes on $\Omega^{c}$. 


\section{Null QUADRATURE DOMAins AND harmonic CONTINUATION OF POTENTIALS}

If $u$ is the Schwarz potential of $(\Omega, \mu)$ and $\mathbb{R}^{n} \backslash \operatorname{supp}(\mu)$ is connected, then for any $v \in V[\Omega]$ the function

$$
w(x):= \begin{cases}v(x) & \text { for } x \in \Omega^{c} \\ v(x)-u(x) & \text { for } x \in \Omega\end{cases}
$$

is the real-analytic (harmonic) continuation of the potential $\left.V[\Omega]\right|_{\Omega^{c}}$ across $\partial \Omega$ onto $\Omega \backslash \operatorname{supp}(\mu)$.

In case of null quadrature domains the harmonic continuation $w$ is an entire analytic function and the Schwarz potential satisfies the free boundary problem

$$
\left\{\begin{array}{ll}
\Delta u=-\chi_{\Omega} & \text { in } \mathbb{R}^{n} \\
u=0 & \text { in } \mathbb{R}^{n} \backslash \bar{\Omega}
\end{array} .\right.
$$

Accordingly, if for an open set $\Omega$ the problem (3.1) has a solution $u$, then we call $u$ the Schwarz potential of the domain $\Omega$. The following Proposition relates (3.1) to the global analytic (harmonic) continuation of the external Newtonian potential of $\Omega$.

Proposition 3.1. Let $\Omega=\operatorname{Int}(\bar{\Omega})$ be an open set in $\mathbb{R}^{n}$ and set $D:=$ $R^{n} \backslash \bar{\Omega}$. The following properties of $\Omega$ are equivalent:

(i) $\Omega$ admits the Schwarz potential u;

(ii) for any $v$ in $V[\Omega]$, the external potential $\left.v\right|_{D}$ extends to a harmonic function $w$ in whole space;

(iii) for any $v^{c}$ in $V[D]$, the internal potential $\left.v^{c}\right|_{D}$ extends to a real analytic function $w^{c}$ satisfying the Poisson equation $\Delta w^{c}=-1$ in whole space.

Under any of these conditions,

$$
u=v-w=w^{c}-v^{c} .
$$

is the Schwarz potential of $\Omega$.

Proof. We prove the equivalence (i) $\Leftrightarrow$ (ii) and the first equality in (3.2). The remainder follows from the complementary formula (2.4). If $\left.v\right|_{D}$ extends to an entire harmonic function $w$, then $u:=v-w$ satisfies (3.1) and hence it is the Schwarz potential of $\Omega$. Conversely, if $u$ is the Schwarz potential of $\Omega$, then it follows from (3.1) that the function $w:=v-u$ is harmonic in $\mathbb{R}^{n}$ and equals to $v$ in $D$.

If the Schwarz potential of $\Omega$ is a Newtonian potential, i.e. $u \in V[\Omega]$, then by Proposition 2.5, $\Omega$ is a null quadrature domain. Thus, we have 
the characterization of null quadrature domains in terms of Schwarz potential:

Corollary 3.2 ([22, 35]). An open set $\Omega$ is a null quadrature domain if and only if it admits the Schwarz potential $u$ with

$$
u(x)=O\left(|x|^{2} \log |x|\right), \quad \text { as } x \rightarrow \infty .
$$

We discuss now the place of null quadrature domains in a larger class of domains having the Schwarz potential (3.1). This investigation was initiated by Shapiro in [39]. Note that if $\Omega$ admits a Schwarz potential which does not satisfy (3.3), then $\Omega$ is not a null quadrature domain. Gustafsson and Shapiro [39], and independently Richardson [34], constructed two dimensional domains $\Omega$ with Schwarz potential having any prescribed singularity at infinity. The theorem below extends their results to arbitrary dimension.

Theorem 3.3. There exist domains $\Omega \subset \mathbb{R}^{n}(n \geq 2)$ with the bounded complement and such that their Schwarz potential $u$ has any prescribed type of singularity at infinity, i.e. for any $m>2$ and for any harmonic polynomial $h_{m}$ of degree $m$ there are $\Omega$ with $u(x)=h_{m}(x)+O\left(|x|^{2}\right)$ as $x \rightarrow \infty$; and there are $\Omega$ with $u$ having essential singular point at infinity.

The methods of Gustafsson and Shapiro and of Richardson are based on a perturbation of a disc by special conformal mappings and hence are essentially two-dimensional. In the proof for the general case we use the same idea of the perturbation of a ball, but we reduce the existence of $\Omega$, with the desired properties of the Schwarz potential, to a theorem on the existence "in the small" (i.e. near to a given domain $\Omega_{0}$ ) of the solution to the inverse problem of potential. The first existence theorem of this type was proved by Sretenskii [43] for the external potential and for $\Omega_{0}$ being a ball. Ivanov [21] extended the theorem of Sretenskii to starlike domains by a modification of the techniques of Lichtenstein from the equilibrium figures theory. For $n=2$ the corresponding results due to Cherednichenko (see references in [10]). Further generalizations were obtained by Prilepko and Isakov $[33,20]$, and they rely on Ivanov's arguments. The existence theorem below for the interior inverse problem of potential is a special case of Corollary 5.1.3 from [20].

Theorem 3.4. Let $D_{0}$ be a bounded domain in $\mathbb{R}^{n}(n \geq 2)$ with Newtonian potential $w_{0}$ and with $\partial D_{0} \in C^{2, \lambda}$. Fix arbitrarily $\varepsilon>0$ and denote by $L_{D_{0}, \varepsilon}$ the set of all functions $w$ satisfying the Poisson equation

$$
\Delta w=-1 \quad \text { in } \quad D_{\varepsilon}:=\left\{x: \operatorname{dist}\left(x, D_{0}\right)<\varepsilon\right\} .
$$


Then for any $r>0$, there is $\delta>0$ such that for $w \in L_{D_{0}, \varepsilon}$ satisfying the condition

$$
\left|w(x)-w_{0}(x)\right|+\left|\nabla w(x)-\nabla w_{0}(x)\right|<\delta \quad \text { on } \partial D_{0},
$$

there is a domain $D$, diffeomorphic to $D_{0}$, having the regular analytic boundary lying in the $r$-neighborhood of $\partial D_{0}$ and such that the interior Newtonian potential of $D$ equals to $\left.w\right|_{D}$. There exists $r_{0}=r_{0}\left(D_{0}, \varepsilon\right)$ such that for $r<r_{0}$ the domain $D$ satisfying the above conditions is unique.

Proof of Theorem 3.3. Let $D_{0}=B_{1}(0)$ be the unit ball. The interior potential of $D_{0}$ is given by the known formula

$$
w_{0}(x)=c_{n}-\frac{|x|^{2}}{2 n}
$$

where $c_{2}=1 / 4$ and $c_{n}=1 / 2(n-2)$ for $n>2$. Let $\varepsilon, r>0$, and choose $\delta$ by Theorem 3.4. Take an arbitrary entire harmonic function $h$ and set

$$
M:=\max _{|x|=1}(|h(x)|+|\nabla h(x)|) .
$$

Then the function

$$
w(x):=c_{n}-\frac{|x|^{2}}{2 n}+\frac{\delta}{2 M} h(x)
$$

is a solution of Poisson equation $\Delta w=-1$ in whole space, satisfying (3.4), so by Theorem 3.4 there is a domain $D$ diffeomorphic to the ball with the interior potential $w$. Denote by $v$ the Newtonian potential of $D$. By Proposition 3.1 we conclude that the function $u:=w-v$ is the Schwarz potential of $\Omega:=\mathbb{R}^{n} \backslash \bar{D}$. If $h$ is a harmonic polynomial of degree $m>2$, then $u(x)=\frac{\delta}{2 M} h(x)+O\left(|x|^{2}\right)$ as $x \rightarrow \infty$. If $h$ is a non-polynomial entire harmonic function, then $u$ has an essential singular point at infinity.

Remark 3.5 (Shapiro [39]). If $\Omega_{k}$ is a domain in $\mathbb{R}^{k}(k<n)$ chosen by Theorem 3.3, then the Schwarz potential of the cylinder $\Omega:=\Omega_{k} \times \mathbb{R}^{n-k}$ has the same properties as that of $\Omega_{k}$, but $\Omega$ has unbounded complement.

The following result of Shapiro [39] (see also Shahgholian [37]) shows that if the complement of $\Omega$ is slightly larger than any cylinder over a bounded domain at infinity, and if the Schwarz potential of $\Omega$ is a tempered distribution, then $\Omega$ is a null quadrature domain.

Proposition 3.6. Suppose $\Omega$ admits the Schwarz potential u, and

(i) $\mathbb{R}^{n} \backslash \bar{\Omega}$ contains balls of arbitrary radius,

(ii) $u$ is a tempered distribution. 
Then $\Omega$ is a null quadrature domain.

Shapiro [39] showed that condition (ii) in Proposition 3.6 is indispensable. He constructed an example where $\Omega$ and $\Omega^{c}$ are "equally large", that is, both contain half-spaces.

Proposition 3.7. There exist domains $\Omega=\left\{\left(x_{1}, \ldots, x_{n-1}, x_{n}\right) \in \mathbb{R}^{n}\right.$ : $\left.x_{n}<f\left(x_{1}, \ldots, x_{n-1}\right)\right\}$ such that

(i) $f$ is a bounded real analytic function in $\mathbb{R}^{n-1}$,

(ii) $\Omega$ admits the Schwarz potential that is not a tempered distribution.

\section{Geometric Properties of Null QUADRATURE Domains}

In this section we prove that the complement of a null quadrature domain is a convex set with analytic boundary. This result was conjectured by H.S. Shapiro and proved by him under some additional conditions [39] (see also [8] and [32]).

The main result of this section is the following:

Theorem 4.1. Let $\Omega$ be a null quadrature domain with Schwarz potential u. Then

(i) there is a positive constant $C$ such that

$$
|u(x)| \leq C(1+|x|)^{2}, \quad x \in \mathbb{R}^{n}
$$

(ii) $u \leq 0$ in $\mathbb{R}^{n}$;

(iii) $\partial^{2} u / \partial e^{2} \leq 0$ in $\mathbb{R}^{n}$ for any unit vector $e$;

(iv) the complement of $\Omega$ is a convex set and $\partial \Omega$ is an analytic surface.

Properties (ii)-(iv) were proved in [8, Theorem II] under the restriction that the Schwarz potential $u$ has a quadratic growth. According to Corollary 3.2, the Schwarz potential of a null quadrature domain has $|x|^{2} \log |x|$ growth at infinity. Thus the principal technical target of this section is the establishment the quadratic growth.

Note that Theorem 4.1 (ii) solves a conjecture which was raised by Shapiro [39] and Shahgholian [37], namely, that any solution $u$ to (3.1) satisfying the growth condition $u(x)=O\left(|x|^{2} \log |x|\right)$ is non-positive.

When the Schwarz potential $u$ is a limiting (global) case of the classical obstacle problem, then $u \leq 0$ in $\mathbb{R}^{n}$ in addition to (3.1). In this case the quadratic growth is a consequence of the boundedness of the second order derivatives and properties (iii) and (iv) follow from the known regularity results for the obstacle problem [5, 6], [14, Ch.2]. 
The proof of Theorem 4.1 will be split into two cases: "thick" and "thin" complement $\left(\mathbb{R}^{n} \backslash \Omega\right)$ at infinity, where the "size" is expressed here by Lebesgue density at infinity.

In the case of thick complement we will prove the expected result, namely, $\Omega$ is a half-space. This means that the boundary $\partial \Omega$ is regular at infinity. We will employ common techniques of free boundaries, such as blow-up and a monotonicity formula combined with Shapiro's quasibalayage [42]. In the singular case of thin complement we will adopt special techniques from [25].

\subsection{Null Quadrature Domains with Thick Complement.}

We denote by $|D|$ the Lebesgue measure of a set $D$ in $\mathbb{R}^{n}$ and $B_{\rho}$ the ball with radius $\rho$ centered at the origin.

Theorem 4.2. A null quadrature domain $\Omega$ satisfying condition

$$
\limsup _{\rho \rightarrow \infty} \frac{\left|\Omega^{c} \cap B_{\rho}\right|}{\left|B_{\rho}\right|}>0
$$

is a half-space.

Lemma 4.3. Let $u$ be the Schwarz potential of null quadrature domain $\Omega$ which satisfies condition (4.1), then there is a positive constant $C$ such that

$$
|u(x)| \leq C(1+|x|)^{2} .
$$

Set

$$
S_{j}=S_{j}(u)=\sup _{B_{2^{j}}}|u|, \quad j=1,2,3, \ldots
$$

Then (4.2) is equivalent to

$$
S_{j} \leq C\left(2^{j}\right)^{2} \quad \text { for all } j \in \mathbb{N} .
$$

We use first Shapiro technique's of quasi-balayage [42] which gives (4.4) along a subsequence of $\left\{2^{j}\right\}$, and then the main difficulty is to extend it to the entire sequence.

Theorem 4.4 (Shapiro $[42, \S 6])$. Let $\sigma \in L^{\infty}\left(\mathbb{R}^{n}\right)$ and $w$ be a tempered distribution on $\mathbb{R}^{n}$ satisfying the system

$$
\begin{cases}\Delta w=-\sigma \chi_{\Omega} & \text { in } \mathbb{R}^{n} \\ w(x)=0 & x \in \mathbb{R}^{n} \backslash \bar{\Omega} .\end{cases}
$$

If

$$
\frac{\left|\Omega^{c} \cap B_{\rho}\right|}{\left|B_{\rho}\right|} \geq c_{0}>0,
$$


then for $|x|=\rho$ there holds

$$
|w(x)| \leq C_{1} \rho^{2}
$$

and the constant $C_{1}$ depends on $c_{0},\|\sigma\|_{L^{\infty}}$ and the dimension.

Proposition 4.5. Retaining the hypotheses of Lemma 4.3, then there is an infinite subset $\mathbb{K}$ of the natural numbers such that (4.4) holds for $j_{k} \in \mathbb{K}$.

Proof. Assuming (4.1), then there is a sequence $\left\{\rho_{k}\right\}$ tending to infinity and a positive constant $c_{0}$ such that (4.6) holds. We may apply now Theorem 4.4 to $u$, the Schwarz potential of $\Omega$, then inequality (4.7) together with the fact that $|u|$ is subharmonic yields that

$$
\sup _{B_{\rho_{k}}}|u|=\sup _{\left\{|x|=\rho_{k}\right\}}|u| \leq C_{1} \rho_{k}^{2} .
$$

Since for each $k$ there is an integer $j_{k}$ such that $2^{j_{k}} \leq \rho_{k} \leq 2^{j_{k}+1}$, we get

$$
S_{j_{k}} \leq 4 C_{1}\left(2^{j_{k}}\right)^{2}
$$

for infinitely many $j_{k}$.

Thus Lemma 4.3 follows from:

Lemma 4.6. Let $u$ be the Schwarz potential of null quadrature domain $\Omega$ and defined $S_{j}$ by (4.3). If there is an infinite subset $\mathbb{K}$ of the natural numbers such that

$$
S_{j} \leq C_{2}\left(2^{j}\right)^{2} \quad \text { for } j \in \mathbb{K}
$$

then there is a constant $C_{0}$ so that

$$
S_{j} \leq 4 C_{0}\left(2^{j}\right)^{2} \quad \text { for all positive } j .
$$

In the proof of this lemma we will use Alt, Caffarelli and Friedman's monotonicity formula [3].

Theorem 4.7 ([3]). Suppose $v_{1}$ and $v_{2}$ are non-negative subharmonic functions in $B_{R}$ such that $v_{1}(x) v_{2}(x)=0$ in $B_{R}$ and $v_{1}(0)=v_{2}(0)=0$. Then for $0<r<R$,

$$
\Phi(r):=\Phi\left(r, v_{1}, v_{2}\right)=\frac{1}{r^{4}}\left(\int_{B_{r}} \frac{\left|\nabla v_{1}\right|^{2}}{|x|^{n-2}} d x\right)\left(\int_{B_{r}} \frac{\left|\nabla v_{2}\right|^{2}}{|x|^{n-2}} d x\right) .
$$

is a nondecreasing function of $r$. 
Let us make several remarks about the applications of this theorem here. We may assume the origin belongs to $\Omega^{c}$. Then the partial derivatives $\partial_{i} u$, of the Schwarz potential $u$ of $\Omega$, are harmonic in $\Omega$ and vanish on $\mathbb{R}^{n} \backslash \Omega$. Therefore $\partial_{i} u^{+}:=\max \left\{\partial_{i} u, 0\right\}$ and $\partial_{i} u^{-}:=-\min \left\{\partial_{i} u, 0\right\}$ are subharmonic and satisfy the requirements of Theorem 4.7. Further properties are:

a) If the second derivatives of $u$ are bounded by $K$ in $B_{r}$, then

$$
\Phi\left(r, \partial_{i} u^{+}, \partial_{i} u^{-}\right) \leq \frac{(n K)^{4} \omega_{n}^{2}}{4} .
$$

b) $\Phi$ is homogeneous with respect to the scaling $u_{\rho}(x)=u(\rho x) / \rho^{2}$, that is,

$$
\Phi\left(r \rho, \partial_{i} u^{+}, \partial_{i} u^{-}\right)=\Phi\left(r, \partial_{i} u_{\rho}^{+}, \partial_{i} u_{\rho}^{-}\right) .
$$

Proof of Lemma 4.6. Let $\mathbb{M}$ be the set of all positive integers such that

$$
4 S_{j-1}>S_{j} .
$$

We consider first the case when $\mathbb{M}$ is finite. Then there is $j_{0} \in \mathbb{K}$ such that

$$
S_{j} \leq \frac{1}{4} S_{j+1} \quad \text { for all } j \geq j_{0} .
$$

Since $\mathbb{K}$ is infinite, for each such $j$ there is $j_{k} \in \mathbb{K}$ such that $j<j_{k}$. Let $m=j_{k}-j$, then by (4.9) and (4.14),

$$
S_{j} \leq \frac{S_{j+m}}{4^{m}}=\frac{S_{j_{k}}}{4^{m}} \leq C_{2} \frac{\left(2^{(j+m)}\right)^{2}}{4^{m}}=C_{2}\left(2^{j}\right)^{2} \quad \text { for all } j \geq j_{0} .
$$

For $j<j_{0}, S_{j} \leq S_{j_{0}} \leq C_{2} 2^{2\left(j_{0}-j\right)}\left(2^{j}\right)^{2}$. Thus (4.10) holds for all $j$.

In the case where $\mathbb{M}$ is infinite, we will prove that there is a positive constant $C_{0}$ such that

$$
S_{j} \leq C_{0}\left(2^{j}\right)^{2} \quad \text { for all } j \in \mathbb{M} .
$$

Then inequality (4.10) follows from (4.15). Indeed, if not, then there is $j_{1}$ such that $S_{j_{1}}>4 C_{0}\left(2^{j_{1}}\right)^{2}$. Since $\mathbb{M}$ is infinite, there is an integer $j_{2} \in \mathbb{M}$ such that $j_{1}<j_{2}$. Let

$$
j_{3}=\max \left\{j<j_{2}: 4 C_{0}\left(2^{j}\right)^{2}<S_{j}\right\} .
$$

Then

$$
S_{\left(j_{3}+1\right)} \leq 4\left(4 C_{0}\left(2^{j_{3}}\right)^{2}\right)<4 S_{j_{3}} .
$$

Hence $j_{3}+1 \in \mathbb{M}$, so by (4.15),

$$
4 C_{0}\left(2^{j_{3}}\right)^{2}<S_{j_{3}} \leq S_{\left(j_{3}+1\right)} \leq C_{0}\left(2^{\left(j_{3}+1\right)}\right)^{2}=4 C_{0}\left(2^{j_{3}}\right)^{2}
$$

and this yields a contradiction. 
Thus it remains to prove inequality (4.15). We will assume it does not hold and derive a contradiction. So assume there is infinite sequence $\left\{j_{m}\right\} \subset \mathbb{M}$ such that

$$
S_{j_{m}} \geq m\left(2^{j_{m}}\right)^{2}
$$

and set

$$
u_{m}(x)=\frac{u\left(2^{j_{m}} x\right)}{S_{j_{m}-1}} .
$$

This sequence has the following properties: Since $0 \in \Omega^{c}, u_{m}(0)=$ $\left|\nabla u_{m}(0)\right|=0$ for all $m$;

$$
\sup _{B_{(1 / 2)}}\left|u_{m}\right|=1
$$

from (4.13),

$$
\sup _{B_{1}}\left|u_{m}\right|=\frac{S_{j_{m}}}{S_{j_{m}-1}} \leq 4
$$

and from (4.16)

$$
\left|\Delta u_{m}(x)\right|=\left|\frac{2^{2 j_{m}}}{S_{j_{m}-1}} \chi_{\Omega}\left(2^{j_{m}} x\right)\right| \leq \frac{4}{m} .
$$

Applying elliptic estimates, we get that $\left\{u_{m}\right\}$ is bounded in $C^{1, \alpha}\left(B_{(3 / 4)}\right)$ and therefore exists a function $u_{\infty}$ such that $u_{m} \rightarrow u_{\infty}$ and $\nabla u_{m} \rightarrow$ $\nabla u_{\infty}$ uniformly in $B_{(1 / 2)}$ (we do not distinguish between sequences and subsequences).

We invoke now Theorem 4.7. Since $\mathbb{K}$ is infinite, for each $j_{m} \in \mathbb{M}$ there is $j_{k} \in \mathbb{K}$ such that $j_{m} \leq j_{k}$. So by the homogeneity and the monotonicity of $\Phi$ we have,

$$
\begin{aligned}
& \left(\frac{S_{j_{m}-1}}{\left(2^{j_{m}}\right)^{2}}\right)^{4} \Phi\left(\frac{1}{2}, \partial_{i} u_{m}^{+}, \partial_{i} u_{m}^{-}\right) \\
= & \Phi\left(\frac{1}{2} 2^{j_{m}}, \partial_{i} u^{+}, \partial_{i} u^{-}\right) \leq \Phi\left(\frac{1}{2} 2^{j_{k}}, \partial_{i} u^{+}, \partial_{i} u^{-}\right) .
\end{aligned}
$$

In order to bound $\Phi\left(\frac{1}{2} 2^{j_{k}}, \partial_{i} u^{+}, \partial_{i} u^{-}\right)$we set

$$
u_{k}(x)=\frac{u\left(2^{j_{k}} x\right)}{\left(2^{j_{k}}\right)^{2}},
$$

when $j_{k} \in \mathbb{K}$. Then $u_{k}$ satisfies the equations

$$
\left\{\begin{array}{ll}
\Delta u_{k}=1, & \text { in } \Omega_{k} \cap B_{1} \\
u_{k}(x)=\left|\nabla u_{k}(x)\right|=0, & \text { on } B_{1} \backslash \Omega_{k}
\end{array},\right.
$$


where $\chi_{\Omega_{k}}(x)=\chi_{\Omega}\left(2^{j_{k}} x\right)$. So by condition (4.9), $\sup _{B_{1}}\left|u_{k}\right| \leq C_{2}$. Therefore [8, Theorem I] yields that $\left\|u_{k}\right\|_{C^{1,1}\left(B_{(1 / 2)}\right)} \leq C$ and the constant $C$ is independent of $k$. Using properties a) and b) below Theorem 4.7 , we get that

$$
\Phi\left(\frac{1}{2} 2^{j_{k}}, \partial_{i} u^{+}, \partial_{i} u^{-}\right)=\Phi\left(\frac{1}{2}, \partial_{i} u_{k}^{+}, \partial_{i} u_{k}^{-}\right) \leq C .
$$

The combination of the above inequality with (4.13), (4.16) and (4.20) implies that

$$
\begin{aligned}
& \left(\frac{1}{2}\right)^{2 n}\left(\int_{B_{(1 / 2)}}\left|\nabla \partial_{i} u_{m}^{+}\right|^{2} d x\right)\left(\int_{B_{(1 / 2)}}\left|\nabla \partial_{i} u_{m}^{-}\right|^{2} d x\right) \\
& \leq \Phi\left(\frac{1}{2}, \partial_{i} u_{m}^{+}, \partial_{i} u_{m}^{-}\right) \leq\left(\frac{\left(2^{j_{m}}\right)^{2}}{S_{j_{m}-1}}\right)^{4} \Phi\left(\frac{1}{2} 2^{j_{k}}, \partial_{i} u^{+}, \partial_{i} u^{-}\right) \\
& \leq C\left(\frac{\left(2^{j_{m}}\right)^{2}}{S_{j_{m}-1}}\right)^{4} \leq C\left(\frac{1}{m} \frac{S_{j_{m}}}{S_{j_{m}-1}}\right)^{4} \leq C\left(\frac{4}{m}\right)^{4} .
\end{aligned}
$$

Let $M_{m}^{+}$and $M_{m}^{-}$denote the mean of $\partial_{i} u_{m}^{+}$and $\partial_{i} u_{m}^{-}$over the ball $B_{(1 / 2)}$, respectively. Then Poincaré inequality implies

$$
\begin{aligned}
& \left(\int_{B_{(1 / 2)}}\left|\partial_{i} u_{m}^{+}-M_{m}^{+}\right|^{2} d x\right)\left(\int_{B_{(1 / 2)}}\left|\partial_{i} u_{m}^{-}-M_{m}^{-}\right|^{2} d x\right) \\
\leq & C\left(\int_{B_{(1 / 2)}}\left|\nabla \partial_{i} u_{m}^{+}\right|^{2} d x\right)\left(\int_{B_{(1 / 2)}}\left|\nabla \partial_{i} u_{m}^{-}\right|^{2} d x\right) \leq C\left(\frac{4}{m}\right)^{4} .
\end{aligned}
$$

Letting $m$ tends to infinity, we get

$$
\left(\int_{B_{(1 / 2)}}\left|\partial_{i} u_{\infty}^{+}-M_{\infty}^{+}\right|^{2} d x\right)\left(\int_{B_{(1 / 2)}}\left|\partial_{i} u_{\infty}^{-}-M_{\infty}^{-}\right|^{2} d x\right)=0 .
$$

Since $\partial_{i} u_{\infty}^{ \pm}(0)=0$, equality (4.22) implies that $\partial_{i} u_{\infty}$ does not change sign in $B_{(1 / 2)}$. In addition, (4.19) shows that $\partial_{i} u_{\infty}$ is harmonic in $B_{(1 / 2)}$, so the maximum principle implies that $\partial_{i} u_{\infty} \equiv 0$ for $i=1, \ldots, n$. Thus $u_{\infty}$ identically equals to a constant and since $u_{\infty}(0)=0$, we conclude that $u_{\infty} \equiv 0$ in $B_{(1 / 2)}$. But this contradicts (4.17). This completes the proof of Lemma 4.6. 
Having the quadratic growth at hand (Lemma 4.3), we can now follow the proof of [8, Theorem II, Case 2] and conclude that if $\Omega$ is a null quadrature domain satisfying condition (4.1), then it is a half-space. But we have chosen here a different approach which is based upon the computation of a potential of a cone (2.5). This method requires a precondition that the Schwarz potential is non-positive, therefore in the case of a global obstacle problem we may use it without relying on [8].

Proof of Theorem 4.2. The Schwarz potential $u$ is non-positive since it has quadratic expression growth, therefore by [6, Corollary 7], $\mathbb{R}^{n} \backslash \Omega$ is convex. Let $\left\{\rho_{k}\right\}$ be a sequence tending to infinity and set

$$
u_{k}(x):=\frac{u\left(\rho_{k} x\right)}{\rho_{k}^{2}} .
$$

Then inequality (4.2) implies that $u_{k}$ converges to a function $u_{\infty}$ and $\nabla u_{k}$ converges to $\nabla u_{\infty}$ uniformly on compact subset of $\mathbb{R}^{n}$. Let $K:=$ $\left\{x: u_{\infty}(x)<0\right\}$, then $\Delta u_{\infty}=-1$ on $K, u_{\infty}$ vanishes on complement $K^{c}$ and $K^{c}$ is also convex. Without loss of generality we may assume the origin belongs to $\partial \Omega$ which implies that $0 \in \partial K$. By means of [26, Theorem 2.5], $K$ is a cone and therefore $u_{\infty}$ is a potential of the cone $K$. So $u_{\infty}$ has the formula (2.5) and since $u_{\infty} \leq 0$ in $\mathbb{R}^{n}$, the expression (2.5) cannot contain the logarithmic term. In addition $K^{c}$ is convex, so Corollary 3.9 in [25] implies that $K$ is half-space.

We will accomplish the proof by showing that

$$
K^{c} \subset \Omega^{c} .
$$

Since $\Omega^{c}$ is convex and $K^{c}$ is a half-space, the above inclusion implies that $\Omega$ must be a half space. Note that both $K^{c}$ and $\Omega^{c}$ are closed sets, hence it suffices to show $\operatorname{int}\left(K^{c}\right) \subset \operatorname{int}\left(\Omega^{c}\right)$. Suppose it fails, then there is $x_{0} \in \operatorname{int}\left(K^{c}\right)$ such that $x_{0} \in \bar{\Omega}$. Since $\Omega^{c}$ is convex, $\rho x_{0} \in \bar{\Omega}$ for all $\rho \geq 1$. Let $r$ be a positive number so that $B_{r}\left(x_{0}\right) \subset \operatorname{int}\left(K^{c}\right)$, then by Caffarelli's Lemma (see e.g. [6, Lemma 5]),

$$
\inf _{B_{r \rho}\left(\rho x_{0}\right)} u(x) \leq-c(r \rho)^{2}
$$

for any $\rho \geq 1$ and where $c$ is a positive constant. Hence $\inf _{B_{r}\left(x_{0}\right)} u_{k}(x) \leq$ $-c r^{2}$ for all $k$. Since $u_{k}$ converges uniformly to $u_{\infty}$, we have obtained that $B_{r}\left(x_{0}\right) \not \subset \operatorname{int}\left(K^{c}\right)$ which is a contradiction. This establish (4.23) and completes the proof of Theorem 4.2.

\subsection{Null Quadrature Domains with Thin Complement.}


In this section we will prove the quadratic growth of the Schwarz potential of null quadrature $\Omega$ under the complementary condition to (4.1), namely

$$
\lim _{\rho \rightarrow \infty} \frac{\left|\Omega^{c} \cap B_{\rho}\right|}{\left|B_{\rho}\right|}=0
$$

The main idea here is to show that if a set $D$ has zero Lebesgue density at infinity, then the blow-up of its potential will tend to zero. Using the representation (3.2) of the Schwarz potential, we will get that the blow-up of the Schwarz potential tends to a quadratic polynomial and that yields the quadratic growth in this case.

Proposition 4.8. If $D$ is set satisfying the condition

$$
\lim _{\rho \rightarrow \infty} \frac{\left|D \cap B_{\rho}\right|}{\left|B_{\rho}\right|}=0
$$

then

$$
\lim _{\rho \rightarrow \infty}\left\|\chi_{D}(\rho \cdot)\right\|_{\mathcal{L}}=0
$$

where the norm $\|\cdot\|_{\mathcal{L}}$ is defined by (2.2).

Proof. Making a change of the variables, we have

$$
\int_{B_{R}} \chi_{D}(\rho x) d x=R^{n} \frac{\left|B_{(\rho R)} \cap D\right|}{\left|B_{(\rho R)}\right|} \rightarrow 0 \quad \text { as } \rho \rightarrow \infty .
$$

For a given $\epsilon>0$, we may find $R>0$ such that

$$
\int_{\{R \leq|x|\}} \frac{\chi_{D}(\rho x)}{(1+|x|)^{n+1}} d x<\epsilon
$$

for all $\rho$. Hence,

$$
\begin{aligned}
\left\|\chi_{D}(\rho \cdot)\right\|_{\mathcal{L}} & =\int_{B_{R}} \frac{\chi_{D}(\rho x)}{(1+|x|)^{n+1}} d x+\int_{\{R \leq|x|\}} \frac{\chi_{D}(\rho x)}{(1+|x|)^{n+1}} d x \\
& \leq \int_{B_{R}} \chi_{D}(\rho x) d x+\epsilon \rightarrow \epsilon \quad \text { as } \rho \rightarrow \infty .
\end{aligned}
$$

Proposition 4.9. Let $V_{2}(D)$ be the potential that is defined by (2.7). If a set $D$ satisfies condition (4.26), then

$$
\lim _{\rho \rightarrow \infty} \frac{1}{\rho^{2}} V_{2}(D)(\rho x)=0
$$

uniformly on compact subsets of $\mathbb{R}^{n}$. 
Proof. Let $\phi_{\rho}$ be a cut-off function such that $\phi_{\rho}(x)=1$ for $|x| \leq \rho$, $\phi_{\rho}(x)=0$ for $|x| \geq 2 \rho$ and $\left|\partial^{\alpha} \phi_{\rho}(x)\right| \leq C \rho^{-|\alpha|}$, and set

$$
w_{\rho}(x):=\phi_{\rho}(x) \frac{1}{\rho^{2}} V_{2}(D)(\rho x) .
$$

Since $w_{\rho} \in C_{0}^{1}\left(\mathbb{R}^{n}\right), V_{2}\left(\Delta w_{\rho}\right)=w_{p}$ and hence it suffices to show that $V_{2}\left(\Delta w_{\rho}\right)$ tends to zero uniformly. We first claim that $\left\|\Delta w_{\rho}\right\|_{\mathcal{L}} \rightarrow 0$, indeed,

$$
\begin{aligned}
\Delta w_{\rho}(x) & =\phi_{\rho}(x) \chi_{D}(\rho x) \\
& +\frac{2}{\rho}\left\{\nabla \phi_{\rho}(x) \cdot \nabla V_{2}(D)(\rho x)\right\}+\frac{1}{\rho^{2}} \Delta \phi_{\rho}(x) V_{2}(D)(\rho x) .
\end{aligned}
$$

The $\mathcal{L}$-norm of the first term tends to zero by Proposition 4.8 . By a virtue of the growth properties of the potential (2.8) and the properties of the mollifier $\phi_{\rho}$,

$$
\begin{aligned}
& \left|\frac{2}{\rho}\left\{\nabla \phi_{\rho}(x) \cdot \nabla V_{2}\left(\chi_{D}\right)(\rho x)\right\}\right| \\
\leq & C \frac{2 \chi_{\{\rho \leq|x| \leq 2 \rho\}}}{\rho^{2}}(1+|\rho x|) \log (2+|\rho x|) \leq C \log \left(2+\rho^{2}\right)
\end{aligned}
$$

and

$$
\begin{aligned}
& \left|\frac{1}{\rho^{2}} \Delta \phi_{\rho}(x) V_{2}\left(\chi_{D}\right)(\rho x)\right| \\
\leq & C \frac{\chi\{\rho \leq|x| \leq 2 \rho\}}{\rho^{4}}(1+|\rho x|)^{2} \log (2+|\rho x|) \leq C \log \left(2+\rho^{2}\right) .
\end{aligned}
$$

Thus,

$$
\begin{aligned}
& \left\|\frac{2}{\rho}\left\{\nabla \phi_{\rho} \cdot \nabla V_{2}\left(\chi_{D}\right)(\rho \cdot)\right\}+\frac{1}{\rho^{2}} \Delta \phi_{\rho} V_{2}\left(\chi_{D}\right)(\rho \cdot)\right\|_{\mathcal{L}} \\
\leq & C \log \left(2+\rho^{2}\right) \int_{\{\rho \leq|x| \leq 2 \rho\}} \frac{1}{(1+|x|)^{(n+1)}} d x \leq C \frac{\log \left(2+\rho^{2}\right)}{\rho} \rightarrow 0 .
\end{aligned}
$$

Next we proceed in a similar manner to the proof of Theorem 3.12 in [25]. Let $J_{2}(x, y)$ be the kernel that is defined by (2.6). Then for $|x| \leq R$,

$$
\begin{aligned}
& \left|V_{2}\left(\Delta w_{\rho}\right)(x)\right| \leq \int_{\{|y| \leq 1\}} J(x-y) \Delta w_{\rho}(y) d y \\
& +\int_{\{1 \leq|y| \leq 2 R+1\}}\left|J_{2}(x, y) \Delta w_{\rho}(y) d y\right|+\int_{\{2 R+1 \leq|y|\}}\left|J_{2}(x, y) \Delta w_{\rho}(y) d y\right| .
\end{aligned}
$$


Since $D$ has zero Lebesgue density, we see from (4.27) that $\Delta w_{\rho}$ tends to zero almost everywhere in $B_{R}$, so the first two terms tend to zero by Lebesgue dominated theorem, while for the last one we use the estimate

$$
\left|J_{2}(x, y)\right| \leq C \frac{R^{3}}{|y|^{(n+1)}} \quad \text { for }|x| \leq R \text { and } 2 R+1 \leq|y| .
$$

Knowing that $\left\|\Delta w_{\rho}\right\|_{\mathcal{L}} \rightarrow 0$, we get that

$$
\int_{\{2 R+1 \leq|y|\}}\left|J_{2}(x, y) \Delta w_{\rho}(y) d y\right| \leq C R^{3} \int \frac{\left|\Delta w_{\rho}(y)\right| d y}{(1+|y|)^{(n+1)}}=C R^{3}\left\|\Delta w_{\rho}\right\|_{\mathcal{L}}
$$

also tends to zero.

Let $u$ be the Schwarz potential of a null quadrature domain $\Omega$ then by formula (3.2),

$$
u(x)=P(x)-V_{2}\left(\Omega^{c}\right)(x),
$$

where $P=w_{\left.\right|_{\Omega^{c}}}^{c}$ for some $w^{c} \in V\left[\Omega^{c}\right]$. According to Theorem 2.6,

$$
P(x)=\sum_{i, j} a_{i j} x_{i} x_{j}+\sum_{i} b_{i} x_{i}+c .
$$

Applying Proposition 4.9 to $\Omega^{c}$ we get the desired quadratic growth.

Lemma 4.10. Suppose $\Omega$ is a null quadrature domain such that the complement $\Omega^{c}$ satisfies condition (4.26), then

$$
\lim _{\rho \rightarrow \infty} \frac{u(\rho x)}{\rho^{2}}=\sum_{i, j} a_{i j} x_{i} x_{j}
$$

and the convergence is uniform on compact subsets of $\mathbb{R}^{n}$. Consequentially, there is a constant $C$ such that

$$
|u(x)| \leq C(1+|x|)^{2} .
$$

Remark 4.11. It is possible to show the quadratic growth for unbounded quadrature domains of measures with compact support. In that case the monotonicity formula Theorem 4.7 need to be replaced by a monotonicity formula of Caffarelli, Jerison and Kenig [7].

\subsection{The structure of null quadrature domains.}

In this subsection we discuss the relations between shape of null quadrature domains and the quadratic polynomial (4.29), the internal potential of the complement.

We first note that if the complement $\Omega^{c}$ of a null quadrature domain has positive upper Lebesgue density at infinity, then Theorem 4.2 implies that $\Omega$ is a half space. Hence, in an appropriate coordinate system $P(x)=-\frac{1}{2} x_{n}^{2}$. 
In the case of thin complement we conclude from the limit (4.30) and Theorem 4.1 (ii) that:

Corollary 4.12. Suppose $\Omega$ is a null quadrature domain such that the complement $\Omega^{c}$ satisfies condition (4.26), then

$$
\sum_{i, j} a_{i j} x_{i} x_{j} \leq 0
$$

Let $A=\left(a_{i j}\right)$ be the matrix associated with this polynomial. The limit (4.30) identifies with each null quadrature domain with thin complement a unique matrix $A$.

When $\Omega^{c}$ is bounded, then it is an ellipsoid and consequently the matrix $A$ is negative definite (see. e.g. [23]). In addition, up to a translation and dilation, there is one to one corresponding between the class of null quadrature domains having a bounded complement and negative defined matrices.

In case $\Omega^{c}$ is unbounded, it must contain an infinite ray, since by Theorem 4.1 it is a convex set. We may choose a coordinates system so that this ray is the positive $x_{1}$-axis. Applying the limit (4.30) and its first order derivatives along this ray, we conclude that $a_{1, j}=0$, $j=1, \ldots, n$. Thus the matrix $A$ is rank deficient when $\Omega^{c}$ is unbounded.

We use now the following known geometric facts (for the proof see e.g. Appendix of [2])

Lemma 4.13. Let $D \neq \mathbb{R}^{n}$ be an unbounded convex domain in $\mathbb{R}^{n}$. Then $D$ has (at least) one of the following properties:

(i) $D$ is a cylinder;

(ii) $\partial D$ is a graph of a convex function in an appropriate Cartesian coordinate system in $\mathbb{R}^{n}$.

So in the case of unbounded thin complement there are two options for each semi-negative symmetric matrix $A$ with rank $k<n$ :

(a) If the complement is contained in a strip, then it follows from the forthcoming paper [30] (see also [25, Theorem 4.13]) that $\Omega^{c}=E \times \mathbb{R}^{n-k}$, where $E$ is an ellipsoid in $\mathbb{R}^{k}$;

(b) If $\Omega^{c}$ is not contained in a strip, then Lemma 4.13 and Theorem 4.1 imply that $\Omega^{c}=\left\{x_{1}>f\left(x_{n-k+1}, \ldots, x_{n}\right)\right\}$, where $f$ is a convex and real analytic function.

\section{ReFERENCES}

1. D. Aharonov and H.S. Shapiro, Domains on which analytic functions satisfy quadrature identities, J. Analyse Math. 30 (1976), 39-73. 
2. A.D. Alexandrov, Intrinsic geometry of convex surfaces, in A.D. Alexandrov Selected Works, Part II (S.S. Kutateladze, ed.), Taylor \& Francis Group, 2006 [translated from Russian].

3. H.W. Alt, L.A. Caffarelli and A. Friedman, Variational problems with two phases and their free boundaries, Trans. Amer. Math. Soc. 282(2), 431-461.

4. E. DiBenedetto and A. Friedman, Bubble growth in porous media, Indiana Univ. Math. J. 35 (1986), 573-606.

5. L.A. Caffarelli, Compactness methods in free boundary problems, Comm. Partial Diff. Eq. 5 (1980), 427-448.

6. L.A. Caffarelli, The obstacle problem revisited, Jour. Fourier Anal.. Appl. 4 (1998), 383-402.

7. L. A. Caffarelli, D. Jerison and C. E. Kenig, Some new monotonicity theorems with applications to free boundary problems, Ann. of Math. (2) 155 (2002), 369-404.

8. L.A. Caffarelli, L. Karp and H. Shahgholian, Regularity of a free boundary problem with application to the Pompeiu problem, Ann. Math. 151, (2000), 269-292.

9. S. Chandrasekhar, Ellipsoidal Figures of Equilibrium, Dover, 1987.

10. V.G. Cherednichenko, Inverse Logarithmic Potential Problems, VSP BV, 1996.

11. R. Dautray and J. L. Lions, Mathematical Analysis and Numerical Methods for Science and Technology, Volume 1: Physical Origins and Classical Methods, $2^{\text {nd }}$-Edition, Springer, 1990.

12. P. Davis, The Schwarz Function and Its Application, Carus Math. Monographs 17, Math. Assoc. of America, 1974.

13. P. Dive, Attraction des ellipsoides homogénes et rèciproque d'um théorème de Newton, Bull Soc. Math. France 59 (1931),128-140.

14. A. Friedman, Variational Principles and Free Boundary Problems, John Wiley \& Sons, Inc. 1982.

15. A. Friedman and M. Sakai, A characterization of null quadrature domains in $\mathbb{R}^{n}$, Indiana Univ. Math. J. 35 (1986), 607-610.

16. B. Gustafsson, On quadrature domains and on an inverse problem in potential theory, J. Analyse Math. 44 (1990), 172-215.

17. B. Gustafsson and H.S. Shapiro, What is a quadrature domain? In Quadrature Domains and Applications, a Harold S. Shapiro Anniversary Volume. (eds. P. Ebenfelt, B. Gustafsson, D. Khavinson, M. Putinar), Birkhäuser, 2005, p. 1-25.

18. W. K. Hayman, L. Karp and H.S. Shapiro, Newtonian capacity and quasi balayage, Rend. Mat. Appl. 20, no. 7 (2000), 93-129.

19. E. Hölder, Über eine Potentialtheoretische Eigenschaft der Ellipse, Math. Z. 35 (1932), 632-643.

20. V. Isakov, Inverse Source Problems, Amer. Math. Soc. Surveys and Monographs 34, Providence, RI, 1993.

21. V.K. Ivanov, An inverse problem for the potential of a body close to a given one, Izvestiya AN SSSR, Ser: Math. 20 (1956), 793-818 (Russian).

22. L. Karp, Generalized Newtonian potential and its applications, J. Math. Anal. Appl. 174 (1993), 480-497.

23. L. Karp, On the Newtonian potential of ellipsoids, Complex Variables 25 (1995), 367-372. 
24. L. Karp, On Null Quadrature Domains, Computational Methods and Function Theory 8 No. 1, 57-72.

25. L. Karp and A.S. Margulis, Newtonian potential theory for unbounded sources and applications to free boundary problems, J. Analyse Math. 70 (1996), 1-63.

26. L. Karp and H. Shahgholian, Regularity of a free boundary problem near the infinity point, Comm. Partial Differential Equations 25, no. 11-12 (2000), 20552086.

27. L. Karp and H. Shahgholian, On the optimal growth of functions with bounded Laplacian, Electronic Journal of Differential Equations (3)2000 (2000), 1-9.

28. A.S. Margulis, Potential theory for $L^{p}$-densities and its applications to inverse problems of gravimetry, in Theory and Practice of Gravitational and Magnetic Fields Interpretation in USSR, Naukova Dumka Press, Kiev, 1983, pages 188197 (Russian).

29. A.S. Margulis, The moving boundary problem of potential theory, Adv. Math. Sci. Appl. 5(2) (1995), 603-629.

30. A.S. Margulis and L. Karp, Characterization of null quadrature domains with a complement contained in a strip, Preprint.

31. W. Nikliborc, Eine Bemerkung über die Volumpotentiale I, II, Math. Z. 35 (1932), 625-631, 36 (1932), 167-170.

32. B. Ou, Global solutions to a free boundary problem, Comm. P.D.E. 19 (1994), 369-397.

33. A.I. Prilepko, Solvability of the inverse problem of finding a body with variable density with a potential close to the given potential, Siberian Math. J. 11 (1970), 973-981 [translated from Russian].

34. S. Richardson, The characterization of curves by global properties of their Schwarz functions, Complex Variables 15 (1990), 11-17.

35. M. Sakai, Null quadrature domains, J. Analyse Math. 40 (1981), 144-154.

36. M. Sakai, Quadrature Domains, Lecture Notes in Math. 934, Springer-Verlag, Berlin-Heidelberg-New York, 1982.

37. H. Shahgholian, On quadrature domains and the Schwarz Potential, J. Math. Anal. Appl. 177 (1992), 61-78.

38. H.S. Shapiro, Unbounded quadrature domains, in Complex Analysis I, ed. C. Berenstein, Lecture Notes in Math. 1275, Springer-Verlag, Berlin-HeidelbergNew York, 1987, 287-331.

39. H.S. Shapiro, Global geometric aspects of the Cauchy's problem for the Laplace operator, in Geometrical and Algebraical Aspects of Several Complex Variables, 309-324, Cetraro, 1989.

40. H. S. Shapiro, A weighted $L^{1}$ estimate for the Laplace Operator, in Recent Advance in Fourier Analysis and its Applications (Il Ciocco 1989), Kluwer Acad. Publ. Dordrecht 1990, pp. 563-577.

41. H.S. Shapiro, The Schwarz Function and Its Generalization to Higher Dimensions, Univ. of Arkansas Lecture Notes in the Math. 9, Wiley, New York 1992.

42. H.S. Shapiro, Quasi-balayage and a priori estimates for the Laplace operator, Multivariate approximation (Witten-Bommerholz 1996), 203-230, 231-254, Math. Res. 101, Akademie Verlag, Berlin, 1997.

43. L.N. Sretenskii, On an Inverse Problem of Newtonian Potential, Izvestiya Acad. Nauk SSSR, Ser. Mat. 2 (1938), 551-570 (Russian). 
44. L.N. Sretenskii, The Theory of Newtonian Potential, Gostechizdat, Moscow, 1946 (Russian).

E-mail address: karp@braude.ac.il

Department of Mathematics, ORT Braude College, P.O. Box 78, 21982 KARMIEL, ISRAEL

E-mail address: avmirs@yahoo.com

6 Hakozrim St., Herzliya, Israel 